\title{
La actividad de escucha estructurada: un modelo para mejorar la comprensión oral *
}

\author{
Joyce S. Choate
}

Thomas A. Rakes

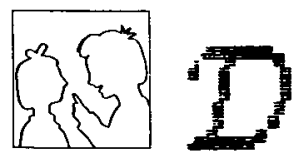

El niño pasa la mayor parte del tiempo de clase escuchando. $¿$ ¿Qué ocurre en esa actividad central? ¿Cómo desarrollarla? Este artículo expone los modelos actuales que intentan responder a esas preguntas y avanza un nuevo planteamiento. Tratar el contenido de la exposición oral como si fuera escrita permite con la misma actividad de escucha resolver dos importantes objetivos: a) mejorar la comprensión lectora de los alumnos y desarrollar las estrategias meta-cognitivas o conscientes de recepción $y, b$ ) al hacerlo, mejorar la comprensión tanto oral como lectora.

A pesar de que muchas publicaciones actuales reconocen a la comprensión oral como un componente integrante del currículum elemental, la mayoría de las indicaciones didácticas que se desprenden se basan en actividades del tipo "cómo hacerlo"; estos tratamientos tienden casi a ignorar elementos tan importantes como el pensamiento localizado, el interrogatorio estructurado, las predicciones, el modelado, la retroalimentación dirigida, y el autocontrol. Tanto si se ha considerado como escucha, audición, comprensión del lenguaje oral o correcto procesamiento del lenguaje, hay poca investigación al respecto.

Pocos pondrían en duda que la escucha forma parte de la metodología de enseñanza. Hace unas tres décadas, Wilt (1950) manifestó que el $60 \%$ de la jornada que el niño pasa en la escuela primaria la dedica a escuchar. Aparentemente, esta práctica no ha cambiado sustancialmente hasta la fe-

*The Structured Listening Activity: A model for improving listening comprehension». The Reading Teacher. Noviembre, 194-199. Reproducido con autorización de Joyce S. Choate y Thomas A. Rakes y la International Reading Association (C. Traducción al castellano, CLEE, 1989 (Traducción de Carmen Trillo. La I.R.A. no se responsabiliza de la adecuación de la traducción).

* *Listening Comprehension* en el original. En algunos lugares hemos optado por comprensión oral para facilitar la legibilidad del texto. Literalmente podría traducirse como «comprensión a través de la escucha» y se sobreentiende que de una exposición habitualmente leída. 
cha. En la actualidad, los estudiantes de los primeros cursos continúan dedicando la porporción más importantes de la jornada escolar a escuchar (Allen, Brown y Yatvin, 1986).

La mayor parte de las estrategias educativas recomendadas para la escucha parecen ser recetas prácticas, sin la suficiente secuenciación o estructura como para asegurar el progreso o la continuidad, y se hace muy necesaria una secuenciación ordenada del proceso de la que puedan hacer uso regularmente los profesores en el contexto de la clase.

La exposición que sigue contiene una breve revisión de los modelos más conocidos sobre instrucción de la escucha, la explicación de un modelo propuesto para su utilización en la clase y algunos resultados preliminares de un estudio sobre la aplicación del modelo de Actividad de Escucha Estructurada (SLA: Structured Listening Activity).

\section{LA RELACION ESCUCHA-LECTURA}

Numerosos estudios han investigado las interrelaciones entre escucha y comprensión lectora. Los resultados han destacado la importancia de la instrucción específica en habilidades de escucha y se han recomendado diversos modos de presentar las actividades de escucha.

\section{La importancia de la instrucción de la escucha}

La importancia de que los niños lean en voz alta ha sido ampliamente reconocida por expertos en educación (Ministerio de Educación de los EE.UU, 1980) e incluso por escritores populares como Erma Bombeck (1986). La escucha permite a los chicos desarrollar la comprensión del lenguaje que es, según Carroll (1977), uno de los elementos esenciales para la comprensión lectora.

Sticht y James (1984), a partir de la revisión que realizaron de la literatura existente, manifestaron que, de doce estudios, diez mostraban una transferencia de aprendizaje exitosa de la escucha de la lectura. Boodt (1984) encontró que la instrucción en la comprensión crítica de la escucha consiguió mejorar la lectura de niños retrasados de cuarto, quinto y sexto curso. Pearson y Fielding (1982) concluyeron que una instrucción específica en comprensión oral podría producir mejoras en la escucha y, en lectores maduros, el entrenamiento en escucha también podría mejorar la comprensión lectora.

\section{Modelos de instrucción más empleados}

Basándose en la creencia de que la instrucción en escucha mejora las habilidades lectoras, se han recomendado diversos e interesantes procedimientos de instrucción. Hay dos tipos básicos:

El método de Impresión Neurológica de Heckleman o NIM (1966, 1969) es una técnica de lectura oral continuada que es utilizada con frecuencia en programas terapéuticos. Más en la línea de esta exposición, el segundo tipo sigue los pasos de la Actividad de Lectura Dirigida (Betts, 1946) o la Actividad Dirigida de Lectura-Pensamiento (Stauffer, 1969). 
La Unidad Didáctica de Transferencia de Escucha-Lectura (Cunningham, 1975) y la Actividad Dirigida Escucha-Pensamiento o DLTA (Stauffer, 1975, 1980) son ejemplos de estos procedimientos.

El modelo de Cunningham presenta actividades paralelas en escucha y en lectura, donde el mismo objetivo específico que se da a los estudiantes para la unidad de escucha es repetido en la lección de la unidad de lectura que sigue a continuación, animando a los estudiantes a que expliquen cómo llegan a sus respuestas en ambas actividades.

El DLTA de Stauffer empieza haciendo que los estudiantes pronostiquen el contenido de un pasaje a partir del título y la ilustración. A medida que avanza la actividad de escuchar, los estudiantes van sintetizando periódicamente y predicen el texto que va a seguir, y por último se organiza una discusión dirigida a volver a contar el pasaje.

La Actividad de Escucha Estructurada o SLA está basada en la concepción general de este tipo de planes de enseñanza, más los componentes claves de la instrucción lectora eficaz, y en los tests correspondientes.

\section{UN FUNDAMENTO PARA LA PRACTICA ADECUADA}

El modelo de Actividad de Escucha Estructurada incluye cinco pasos secuenciados que están respaldados por numerosas investigaciones. Están incorporados todos aquellos aspectos importantes de la instrucción eficaz para la comprensión lectora.

El SLA es un compendio de prácticas que han sido sugeridas de diversas formas en numerosos estudios. La premisa que subyace al DRTA en la estructura del modelo de escucha es que lo que mejora la comprensión lectora puede también mejorar la comprensión de escucha.

Pearson y Fielding (1982) proponen un sistema de comprensión del lenguaje que interrelaciona la escucha, el habla y la lectura. El DRTA viene también avalado por el uso popular y por los datos empíricos (Davidson, 1970; Petre, 1970).

Cada paso del SLA refleja un área de recomendaciones para la enseñanza de la comprensión lectora previamente extraídas de la "práctica adecuada».

Se ha reconocido ampliamente la importancia de elaborar perspectivas, ampliar los esquemas y de formar conceptos (Mc Neil, 1987). En el SLA, estos procedimientos se aplican en la Fase 1 para preparar al oyente.

La necesidad de establecer finalidades, Fase 2, es un procedimiento bien aceptado y documentado (Rothkopf, 1982). La Fase 3, lectura en voz alta, requiere ayudas visuales en la medida en que desarrolla esquemas y proporciona al alumno una base concreta. Se utiliza la estimulación de la formación de imágenes como una ayuda para la consecución de la comprensión (Miccinati, 1982; Pressley, 1977). La predicción dirigida, unạ estrategia verificada (Collins y Smith, 1982; Pearson, 1982 y Fielding, 1982), forma también parte de la fase 3.

La Fase 4 se dedica al cuestionario. Desde Guszak (1967) a Durkin (1978-1979), se ha destacado la necesidad de utilizar adecuadamente el tipo de preguntas y hacerlas con suficiente variedad; Pearson (1982) ofrece una guía para preguntas útiles, explicando la necesidad de algo más que pre- 
guntas literales cargadas de hechos. En el SLA, además de incluir preguntas equilibradas en contenido, se incorporan aspectos metacognitivos al tener que explicar los estudiantes el proceso de respuesta, por lo que desarrollan y comparten conductas de autoevaluación y verificación (Mc Neil, 1987).

La Recitación, fase final en el SLA, conlleva que el estudiante vuelva a narrar, sintetice y elabore mediante el uso de preguntas dirigidas. Así pues, se permite que los estudiantes refieran sus ideas y que incluso desarrollen la capacidad de generar sus propias preguntas (Allison, 1971; Keislar y Stern, 1969; Manzo, 1969; Mc Neil y Donant, 1982; Polincsar y Brown, 1984; Reder, 1980).

El modelo SLA puede utilizarse para formatear la mayor parte de las experiencias de escucha que los profesores normalmente proporcionan a los niños. Es adecuado para enseñar hablidades específicas de comprensión, enseñar conceptos, ampliar contenido o leer lecciones, ampliar experiencias y vocabulario y proporcionar a los estudiantes experiencias positivas y entretenidas con libros.

\section{SECUENCIACION} elegido.

El proceso comienza presentando información introductoria del texto

\section{Fase 1: Formación de Conceptos}

Efectuar una presentación del texto relacionando el contenido con las experiencias de los estudiantes. Recordar o imaginar experiencias con las que se puede comprender mejor el texto. Comentar el vocabulario especial.

\section{Fase 2: Objetivo de Escucha}

A modo de juego mental proporcione a los estudiantes una directriz para la escucha de un punto o puntos importantes. Sin embargo, evite revelar el resultado en las historias.

\section{Fase 3: Lectura en voz alta}

- Ayudas visuales: Utilice diversas ayudas visuales (dibujos, transparencias, flanenogramas) durante la lectura o narración del texto. Para facilitar a los estudiantes que sigan el texto, centren su atención y refuercen conceptos.

- Claves predictivas: Inserte al menos tres claves de predicción como puntos claves para guiar el pensamiento de los estudiantes y mantener su atención y su participación activa. Las claves podrían incluir: ¿Qué crees que podría ocurrir a continuación? ¿Qué dirá ella? ¿Qué dirá probablemente? ¿Por qué?

\section{Fase 4: Preguntas}

Utilice tres niveles de preguntas para guiar, discutir y evaluar la comprensión de escucha de los estudiantes; equilibre el número de preguntas 
literales importantes con un número igual de preguntas críticas o interpretativas. (Para un pasaje narrativo de quince minutos, el balance de preguntas de comprensión podría ser de cuatro-seis literales importantes, tres-cuatro interpretativas, dos críticas.)

Entremezcle o distancie las preguntas, pidiendo a los estudiantes que expliquen sus respuestas. Cuando se insertan preguntas, éstas hacen pensar y proporcionan claves atencionales. Las preguntas pospuestas se pueden utilizar para la evaluación del profesor, el autocontrol y evaluación de los estudiantes y como estímulos para ulteriores discusiones o aclaraciones.

\section{Fase 5: Recitación}

Guíe a los estudiantes para que resuman el texto. También puede utilizarse el volver a narrar o la elaboración de ideas.

Si los estudiantes tienen dificultad en resumir, la re-narración dirigida es particularmente efectiva. En este caso, haga que a la re-narración sigan preguntas directas que den como resultado un resumen.

Las preguntas encabezadas por «quién, qué, por qué, cuándo» pueden utilizarse para ayudar al estudiante a generar preguntas. Una vez que los estudiantes se familiarizan con el formato, se pueden añadir estrategias como el «InQuest» (Shoop, 1986) para una mayor variedad y participación interactiva adicional.

\section{ANALISIS Y APLICACION DEL SLA}

El SLA conserva muchos elementos de las actividades con las que los profesores de lectura están más familiarizados. La primera fase de lo que podría ser una actividad de lectura dirigida típica o de una lección de lectura dirigida se refleja en la fases primera y segunda del SLA. Se recupera o elabora la información anterior y se da una consigna al oyente para que concentre su atención en puntos importantes del texto.

Las fases segunda y tercera del DRA de Betts, lectura dirigida oral y silenciosa, respectivamente, son análogas a la fase tercera del SLA y a cualquiera de las preguntas de la fase cuarta que se encuentran entremezcladas en el texto. Las predicciones del pasaje en la fase tercera, semejantes a los del DRTA y DLTA de Stauffer, son las que los profesores con experiencia utilizan con frecuencia para mantener la atención de los estudiantes.

Las preguntas realizadas antes, durante y después y las respuestas registradas de la fase cuarta advierten al profesor de las necesidades de instrucción específica de los estudiantes. La fase quinta proporciona la oportunidad de ensayar, revisar o ampliar ideas, una estrategia respetada durante mucho tiempo por los profesores veteranos.

La historia familiar de «Los Tres Ositos» aparece formateada en la Figura 1 de acuerdo con el modelo SLA, donde se marca cada fase del modelo a medida que aparece. El propósito de estructurar la historia de esta manera es estructurar de forma similar el pensamiento, participación y comprensión de los oyentes. 


\section{VERIFICACION EMPIRICA}

La utilización del modelo SLA como medio de mejorar la comprensión lectora y de escucha requiere una investigación exhaustiva que la valide. Hasta el momento, la única evidencia de la efectividad del modelo es el respaldo teórico de la literatura y el progreso manifestado por unos cien estudiantes.

Durante los tres últimos años, los estudiantes universitarios matriculados en un curso práctico de lectura (Reading Practicum), han seguido el método SLA para enseñar comprensión de escucha a niños entre preescolar y

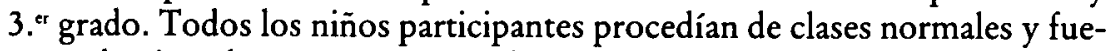
ron seleccionados porque necesitaban instrucción adicional en lectura.

El modelo SLA se utilizó con cada niño durante veinte minutos en cada una de las dos lecciones semanales durante un período de cinco semanas (un total de diez lecciones). Aunque varios cientos de niños fueron instruidos de esta forma, sólo se administró una prueba a aquéllos que habían obtenido en el pretest una lectura oral y silenciosa por debajo del primer nivel. Por tanto, sólo se dispone de datos pre y postest de sesenta y siete sujetos de los cursos K-3.

En cada caso, los sesenta y siete niños fueron evaluados antes y después utilizando textos paralelos de «Procedimientos de Evaluación Individual en Lectura», un inventario informal de habilidades de lectura y escucha.

De acuerdo con las tablas de puntuación de este test, la ganancia promedio de los niños en comprensión de escucha se estimó en un curso completo. Dado que la duración del tratamiento fue ligeramente inferior a tres meses, estas ganancias sobrepasaron el progreso esperado en comprensión lectora. Sin embargo, a causa de la imprecisión de la medida y a la ausencia de un grupo control, las conclusiones sobre la naturaleza y extensión del progreso en comprensión oral deben permanecer en suspenso.

Aparte de la inesperada mejora en comprensión oral, las actividades de escucha proporcionaron una experiencia de aprendizaje positiva. Tanto los profesores como los niños manifestaron reacciones favorables a las actividades. La conducta y atención del estudiante mejoraron notablemente durante el período SLA de cada lección. Los profesores informaron constantemente de niños más tranquilos y felices tanto durante como después de la actividad.

La ganancia en las puntuaciones que se refleja en la prueba informal fue desproporcionadamente elevada. ¿Habían recibido estos niños instrucción anterior en habilidades de escucha? Al ser preguntados, los profesores de los niños de clases normales, afirmaron que leían en voz alta a sus estudiantes diariamente. Manifestaron que usaban frecuentemente libros con vinetas que mostraban a los niños mientras leían. Varios profesores mencionaron que hacian preguntas tras la lectura de la historia, pero sólo ocasionalmente se ofrecía la escucha.

Al parecer se había proporcionado a los niños oportunidades de escucha normales, pero no instrucción específica en comprensión de escucha.

Por tanto, es probable que la ausencia de instrucción sistemática previa en habilidades de escucha puede haber inflado las puntuaciones de rendimiento en escucha de los niños que participaron en el curso práctico de lectura. Otros factores como el efecto Hawthorne y la influencia global de la 


\section{5}

FiguRA 1

\section{SLA en acción: La hisţoria de los Tres Ositos}

Materiales: Copia de la historia y siete ayudas Visuales: V1-3 Osos; V4 Ricitos de oro; V5 papilla; V6 silla; V7 cama.

1. Formación de conceptos

¿Quién es la persona más grande de tu familia? ¿y la siguiente? ¿y quién es el más pequeno? ¿Has visto alguna vez un oso? ¿Dónde? Describe el oso. Esta es una historia sobre una familia de osos: El enorme papá oso; la mediana mamá osa; y el pequeño osito.

2. Objetivos de la escucha

Escucha y decide qué cosas hacen bien la chica y los osos de esta historia y qué hacen mal.

3. Lectura en voz alta

[Utilice efectos visuales $(v)$, señales de predicción $(P)$ y preguntas de comprensión opcionales entremezcladas $(*)$ en los puntos indicados de la lectura. Nota: el mejor lugar para las preguntas destacadas - durante después de la lectura de la historia- depende de la naturaleza y tamaño del grupo de oyentes.]

\begin{tabular}{|c|c|}
\hline Resumen de la historia & Efectos visuales, predicciones y preguntas \\
\hline $\begin{array}{l}\text { Tres osos }{ }^{\mathrm{v}} \text { van de paseo. } \\
\text { Ricitos de Oro entra en la casa }(\mathrm{P})\left(^{*}\right)\end{array}$ & $\begin{array}{l}\text { (v) V } 1-3 \\
\text { (v) V } 4 \text { ¿Qué piensas que hará? } \\
\text { * ¿Qué deberia hacer? }\end{array}$ \\
\hline $\begin{array}{l}\text { Prueba la papillav: se come la papilla del osito. } \\
\text { Prueba las sillas de los osos }{ }^{v} \text { : La de papá es demasiado dura; } \\
\text { la de mamá demasiado blanda, prueba la del osito y ...(P) } \\
\text { La silla se rompe }\left(^{*}\right)\end{array}$ & $\begin{array}{l}\text { (v) V5 } \\
\text { (v) V6 } \\
\text { ¿Por qué crees que la silla se rom- } \\
\text { pió? ¿Cómo lo sabes? }\end{array}$ \\
\hline $\begin{array}{l}\text { Sube al dormitorio. Prueba las camas", } \\
\text { prueba la del osito y ... (P) }\end{array}$ & 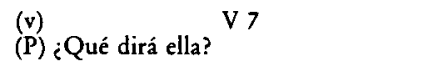 \\
\hline $\begin{array}{l}\text { Los } \operatorname{osos}^{v} \text { vuelven y descubren la papilla } \\
\text { Los osos comprueban las sillas }{ }^{v}\left({ }^{*}\right)\end{array}$ & $\begin{array}{l}\text { (v) V 1-3; V 5; (P) ¿Qué dirá el osito? } \\
\text { (v) V 6; ¿Qué ha pasado a la silla del } \\
\text { osito? }\end{array}$ \\
\hline $\begin{array}{l}\text { Los osos suben, miran las camasv y } \\
\text { encuentran a Ricitos de Orov } \\
\text { Ricitos de Oro se despierta y se escapa }(")\end{array}$ & $\begin{array}{l}\text { (v) V } 7 \\
\text { (v) V } 4 \\
\text { 7 ¿Por qué se escapa Ricitos de Oro? } \\
\text { ¿Qué podria haber pasado si no hu- } \\
\text { biera corrido? }\end{array}$ \\
\hline
\end{tabular}

4. Interrogación (después)

A) Preguntas de comprensión literal

1. ¿Por qué dieron un paseo los osos?

2. ¿Qué es lo que primero probó Ricitos de Oro en la casa de los osos?

3. ¿Qué problemas encontró Ricitos de Oro con la papilla del papá y de la mamá?

4. ¿Qué pasaba con las sillas de papá y de mamá?

*5. ¿Qué pasó con la silla del osito?

6. Cuenta cómo Ricitos de Oro estropeó tres de las pertenencias del osito.

B) Preguntas de comprensión interpretativa

1. ¿Por qué crees que Ricitos de Oro entró en la casa de los osos? ¿Qué te hace pensar así?

*2. ¿Qué debería haber hecho Ricitos de Oro cuando vio que no había nadie en casa?

*3. ¿Por qué crees que se rompió la silla del osito? ¿Cómo lo sabes?

4. ¿Por qué pensaba Ricitos de Oro que las cosas del osito eran adecuadas para ella?

*5. ¿¿Por qué se escapó Ricitos de Oro? ¿Qué podría haber pasado si no hubiera corrido?

C) Preguntas de comprensión crítica

1. ¿Qué partes de esta historia podrian pasar realmente? ¿Qué partes son verosímiles? ¿Cómo lo sabes?

2. Di por qué piensas que Ricitos de Oro era una niña buena o mala. Di por qué los osos eran buenos o malos.

\section{Recitación}

Vuelve a contar rápidamente esta historia. [Haga que los estudiantes verbalicen las lecciones que han aprendido mediante Ricitos de Oro y los osos.] 
instrucción relacionada con la lectura podrían haber inflado también los resultados.

No obstante, las sustanciales ganancias en comprensión de escucha y las respuestas altamente positivas tanto de los profesores como de los ninos nos llevaron a formalizar el modelo SLA y abogan por la realización de más investigaciones.

\section{Referencias}

Allen, Ronald R.; KenNeth L. Brown, y JoANne YATVIn. Learning Language through Communication: A Functional Perspective. Belmont, CA: Wadsworth, 1986.

Allison, ThOMAS L. "A Comparison of Reinforcement Activities for Listening Comprehension Skills.» Tesis doctoral inédita, Syracuse University, Syracuse, NY, 1971.

BetTS, Emmett A. Foundations of Reading Instruction. Nueva York, NY: America Book Company, $1946^{\circ}$

Bombeck, ERma. «How to Encourage Your Child to Read.» Power of the Printed Word Series. Nueva York NY: International Paper Company, 1986.

BOODT, GLORIA M. "Critical Listeners Become Critical Readers in Remedial Reading Class.» The Reading Teacher, vol. 37 junio, 1984, pp. 390-94.

Carroll, John B. *Developmental Parameters in Reading Comprehension. En Cognition, Curriculum and Comprebension, editado por John T. Guthrie. Newark, DE: International Reading Association, 1977.

Collins, Allan, y EDWARD E. Smith. "Teaching the Process of Reading Comprehension.» In How and How Much Can Intelligence Be Increased? editado por Douglas K. Ditterman y Robert J. Sternberg. Norwood, NJ: Ablex, 1982.

CunNingham, PATRICIA M. «Transferring Comprehension From Listening to Reading." The Reading Teacher, vol. 29 (noviembre 1975), pp. 169-72.

Davidson, JANE L. «The Relationship between Teachers' Questions and Pupils' Responses during a Directed Reading Activity and a Directed Reading Thinking Activity.» Universidad de Michigan, Ann Arbor, ML, 1970.

DURKIN, DOLORES. "What Classroom Observations Reveal about Reading Comprehension.» Reading Research Quarterly, vol. 14, n. ${ }^{\circ}$ (1978-1979), pp. 481-533.

GUSZAK, FRANK J. "Teacher Questioning and Reading". The Reading Teacher, vol. 21 (Diciembre 1967), pp. 227-34.

Heckleman, R. G. "Using the Neurological Impress Remedial Technique." Academic Therapy Quarterly, vol. 1 (Verano 1966), pp. 235-39.

HECKLEMAN, R. G. «A Neurological Impress Method of Remedial Reading Instruction.» Academic Therapy Quarterly, vol. 4 (verano 1969), pp. 277-82.

Individual Evaluation Procedures in Reading. Englewood Cliffs, NJ: Prentice-Hall, 1983.

KEISLAR, EVAN, y CAROLYN STERN The Value of Spoken Response in Teaching Listening Skills to Young Children through Programmed Instruction. ED 027973. Washington, DC: ERIC Document Reproduction Service, 1969.

MANZO, ANTHONY V. "The ReQuest Procedure.» Journal of Reading, vol. 13 (noviembre 1969), pp. 123-26.

MCNeil, John D. Reading Comprehension: New Directions for Classroom Practices, 2." ed. Glenview. IL: Scott, Foresman, 1987.

MCNEIL, JOHN, D., y LISBETH DONANT. "Summarization Strategy for Improving Reading Comprehension." En New Inquiries in Reading Instruction, editado por Jerome A. Niles y Larry A. Harris. Rochester, NY: National Reading Conference, 1982.

Miccinati, JeAnNETTE. «The Influence of a Six-week Imagery Training Program on Children's Reading Comprehension." Journal of Reading Behavior, vol. 14 (verano 1982), pp. 197-203.

PalinCSAR, ANNEMARIE S., y ANN L. BROwN. «Reciprocal Teaching of Comprehension-fostering and Comprehension-monitoring Activities." Cognition and Instruction, vol. 2 (Primavera 1984), pp. 117-75.

Pearson, P. David. «Asking Questions About Stories.» Columbus, OH: Ginn Occasional Papers, 1982.

Pearson, P. David, y LINDA Fielding. «Research Update: Listening Comprehension.» Language Arts, vol. 59 (septiembre 1982), pp. 617-29.

PETER, RIChARD M. «Quantily, Quality and Variety of Pupil Responses during an Opencommunication Strucftured Group Directed Reading Activity and a Closed-comunication Structured Group Directed Reading Activity.* Universidad de Delaware, Newark, DE, 1970.

PRESSLEY, MiChaEL. «Imagery and Children's Learning: Putting the Picture in Developmental Perspective." Review of Educational Research, vol. 47 (otono 1977), pp. 585-622. 
REDER, LYNNE,M. *The Role of Elaboration in the Comprehension and Retention of Prose: A Critical Review.» Review of Educational Research, vol. 50 (verano 1980), pp. 5-53.

ROTHKOPF, ERNST Z. «Adjunct Aids and the Control of Mathemagenic Activities during Purposeful Reading." En Reading Expository Materials, editado por Wayne Otto y Sandra White. Nueva York, NY. Academic Press, 1982.

SHOOP, MARY. "InQuest: A Listening and Reading Comprehension Strategy.* The Reading Teacher, vol. 39 (marzo 1986), pp.670-74.

Stauffer, Russell G. Directing Reading Maturity as a Cognitive Process, Nueva York, NY: Harper and Row, 1969.

Stauffer, Russell G. Directing the Reading-Thinking Process. Nueva York, NY. Harper and Row, 1975.

StAUFFER, RUSSELL, G. The Language-Experience Aproach to the Teaching of Reading, 2nd ed. Nueva York, NY: Harper and Row, 1980.

StichF, Thomas G., y James H. James. "Listening and Reading." In Handbook of Reading Research, editado por P. David Pearson. Nueva York, NY: Longman, 1984, pp. 293-317.

U.S. Department of Education. What Works: Research about Teaching and Learning. Washington, DC: Superintendent of documents, 1986.

WILT, MIRIAM E. *A Study of Teacher Awareness of Listening as a Factor in Elementary Education." Journal of Educational Research, vol. 43 (abril 1950), pp. 626-36.

\section{Datos sobre los autores}

Choate trabaja sobre métodos de lectura y evaluación de Educación Especial en la Universidad del Noreste de Louisiana, Monroe, Louisiana. Rakes enseña lectura en la Universidad del Estado de Memphis, Memphis, Tennessee. Actualmente investigan sobre la utilización de las actividades de escucha para mejorar las babilidades de comprensión.

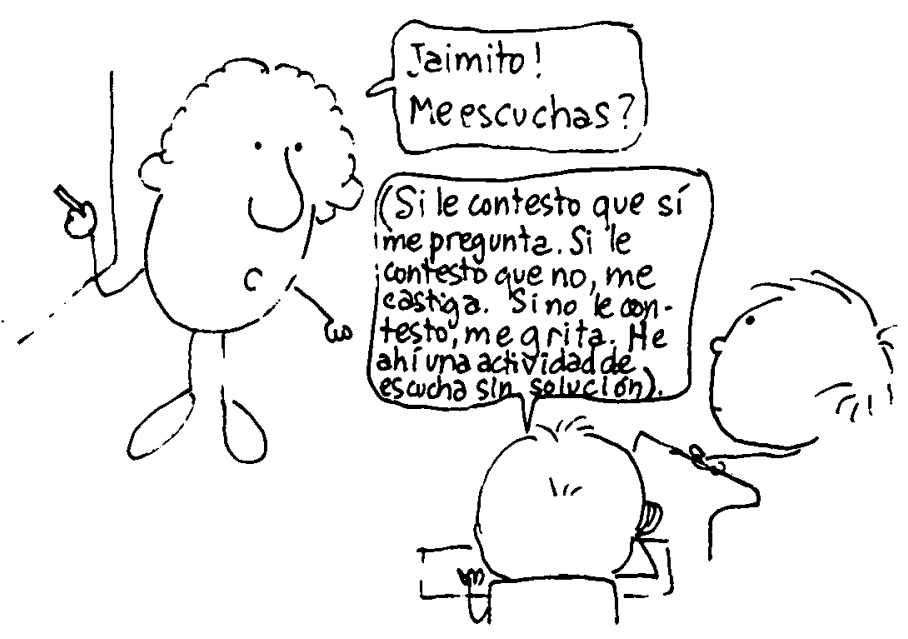

